\title{
SOME EMBEDDING THEOREMS 1
}

KENNETH D. MAGILL, JR.

1. Introduction. It is well known that there exists a homeomorphism between two $Q$-spaces (= realcompact spaces) $X$ and $Y$ if and only if there exists an isomorphism between $C(X)$ and $C(Y)$, their rings of real-valued, continuous functions. This suggests the problem of finding algebraic conditions relating $C(X)$ and $C(Y)$ which are both necessary and sufficient for embedding $Y$ in $X$. Our investigations in this direction have led to the consideration of three types of ring homomorphisms. Before we define these, let us recall that an ideal $M$ of a ring $A$ is a real ideal if $A / M$ is isomorphic to $R$, the field of real numbers. We shall refer to the intersection of a collection of real ideals as a $\delta$-real ideal, and we say that a subring $B$ of a ring $A$ is $\delta$-dense in $A$ if for every pair $M_{1}$ and $M_{2}$ of $\delta$-real ideals of $A$ with $M_{1}-M_{2} \neq \varnothing, M_{1}-M_{2}$ contains an element of $B$.

Definition (1.1). A homomorphism from a ring $A$ into a ring $B$ is a $\delta$-homomorphism if it is nontrivial and the image of $A$ is $\delta$-dense in $B$.

We shall refer to a set of elements of a ring as subreal if it is contained in a real ideal of the ring.

Definition (1.2). A $\delta$-homomorphism is a $\delta F$-homomorphism if the image of every real ideal containing the kernel is subreal.

Definition (1.3). A $\delta$-homomorphism from a ring $A$ into a ring $B$ is a $\delta G$-homomorphism if for every real ideal $M$ of $A$ whose image is subreal, there exists an element $a \notin M$ such that the image of every real ideal not containing $a$ is subreal.

The four main results of this paper are given in Theorems (2.3), (2.5), (2.6) and (2.7). From these it follows that a $Q$-space $Y$ can be embedded in a $Q$-space $X$ if and only if there exists a $\delta$-homomorphism from $C(X)$ into $C(Y)$ and that $Y$ can be embedded in $X$ as a closed (open, dense) subset if and only if there exists a $\delta F$-homomorphism ( $\delta G$-homomorphism, $\delta$-isomorphism) from $C(X)$ into $C(Y)$.

2. The embedding theorems. It will be assumed that all topological spaces discussed here are completely regular and Hausdorff. 1963.

Presented to the Society, February 23, 1963; received by the editors August 9,

1 The results in this paper form a portion of the author's doctoral dissertation written at the Pennsylvania State University under the direction of Professor S. Mrowka, to whom the author wishes to express his appreciation. 
Theorem (2.1). Let $X$ be a $Q$-space, $Y$ an arbitrary space, and $\phi a$ homomorphism from $C(X)$ into $C(Y)$. Then $\phi$ has the property that

(2.1.1) for every $g \in C(Y)$ and $y \notin Z(g),{ }^{2}$ there exists an $f \in C(X)$ such that $y \notin Z(\phi f)$ and $Z(g) \subset Z(\phi f)$

if and only if there is a homeomorphism $h$ from $Y$ into $X$ such that $\phi f=f \circ h$ for all $f \in C(X)$.

Proof. (Sufficiency). Suppose $\phi f=f \circ h$ for all $f \in C(X)$ and that $y \notin Z(g)$ where $g \in C(Y)$. Then $h(y) \notin \mathrm{Cl}_{x}(h[Z(g)])$ and there exists a function $f \in C(X)$ which vanishes on $\mathrm{Cl}_{X}(h[Z(g)])$ but not at $h(y)$. It follows that $y \notin Z(\phi f)$ and $Z(g) \subset Z(\phi f)$.

(Necessity). Now let $\phi$ be a homomorphism from $C(X)$ into $C(Y)$ satisfying (2.1.1). We define a mapping $h$ from $Y$ into $X$ as follows. Let $y \in Y$ be given. Then $\phi_{y}: C(X) \rightarrow R$ is a homomorphism where $\phi_{y}$ is given by $\phi_{y} f=(\phi f)(y)$. Moreover, since $y \notin Z(1),^{3}$ (2.1.1) guarantees the existence of an element $f \in C(X)$ such that $y \notin Z(\phi f)$. Hence $\phi_{y} f \neq 0$, i.e., $\phi_{y}$ is a nontrivial homomorphism into $R$. Since $X$ is a $Q$-space, there exists a unique $x \in X$ such that for each $f \in C(X)$, $\phi_{y} f=f(x)$. We define $h(y)=x$. Then for any $f \in C(X), \phi f=f \circ h$ and it remains for us to show that $h$ is a homeomorphism.

For any $Z$-set $Z(f)$ of $X$, we have $h^{-}[Z(f)]=(f \circ h)^{-}(0)=Z(\phi f)$ and since the $Z$-sets of $X$ form a basis for the closed sets, we conclude that $h$ is continuous.

Now if $y_{1}$ and $y_{2}$ are two distinct elements of $Y$, there is a function $g \in C(Y)$ such that $g\left(y_{1}\right)=0$ and $g\left(y_{2}\right)=1$. Then by (2.1.1) there is an $f \in C(X)$ such that $y_{2} \notin Z(\phi f)$ and $y_{1} \in Z(\phi f)$. Hence $f\left(h\left(y_{2}\right)\right)=\phi f\left(y_{2}\right)$ $\neq 0$ while $f\left(h\left(y_{1}\right)\right)=\phi f\left(y_{1}\right)=0$. Therefore $h\left(y_{1}\right) \neq h\left(y_{2}\right)$ and $h$ is one-toone.

To conclude the proof, we need only show $h^{*}$ is a continuous mapping from $h[Y]$ onto $Y$. Choose $g \in C(Y)$ and $y \notin Z(g)$. Let $f$ denote the function whose existence is guaranteed by (2.1.1). It follows that $h(y) \in h[Y] \cap[X-Z(f)] \subset h[Y-Z(g)]$. Hence $h$ is an open mapping which implies $h^{*}$ is continuous.

If $X$ is any topological space and $F$ is a closed subset of $X$, then $\{f \in C(X): F \subset Z(f)\}$, which we will denote hereafter by $M_{F}$, is an ideal of $C(X)$. Moreover, $M_{F}=\cap\left\{M_{x}: x \in F\right\}$ and hence is a $\delta$-real ideal. In the case of $Q$-spaces, the converse is also true. That is, if $M$ is a $\delta$-real ideal of $C(X)$ where $X$ is a $Q$-space, there is a unique closed subset $F \subset X$ such that $M=M_{F}$. In fact, $X$ is a $Q$-space if

\footnotetext{
$2 Z(g)$ denotes the set of points on which $g$ vanishes and is referred to as a $Z$-set.

- For any real number $k, k$ denotes the function which maps every point of the space into $k$.
} 
and only if every $\delta$-real ideal of $C(X)$ is of the form $M_{F}$ for some closed subset $F$ of $X$. We shall use these facts without explicit mention.

TheOREM (2.2). Let $X$ be an arbitrary topological space and $Y$ a $Q$ space. The following statements concerning a homomorphism $\phi$ from $C(X)$ into $C(Y)$ are equivalent.

(2.2.1) For every $g \in C(Y)$ and $y \notin Z(g)$, there exists an $f \in C(X)$ such that $y \notin Z(\phi f)$ and $Z(g) \subset Z(\phi f)$.

(2.2.2) $\phi$ is a $\delta$-homomorphism.

(2.2.3) The image of $C(X)$ separates points and closed sets and is contained in no $\delta$-real ideal of $C(Y)$.

(2.2.4) $\phi$ is the identity mapping on constant functions and the image of $C(X)$ separates points and closed sets. 4

Proof. (2.2.1) $\Rightarrow(2.2 .2)$. Let $M_{F}$ and $M_{H}$ be two $\delta$-real ideals of $C(Y)$ with $M_{F}-M_{H} \neq \varnothing$. Then $H \nsubseteq F$ and there is an element $y \in H-F$ and a function $g \in C(Y)$ which vanishes on $F$ but not at $y$. According to (2.2.1), there exists an $f \in C(X)$ such that $y \notin Z(\phi f)$ and $Z(g) \subset Z(\phi f)$. Hence $\phi f \in M_{F}-M_{H}$.

(2.2.2) $\Rightarrow(2.2 .3)$. Let $M_{F}$ be any $\delta$-real ideal of $C(Y)$. If $F=Y$, then $M_{F}=(0)$ and since $\phi$ is nontrivial, the image of $C(X)$ cannot be contained in $M_{F}$. On the other hand, if $F \neq Y$, choose $y \notin F$. Then $M_{y}-M_{F} \neq \varnothing$ and hence must contain an element of the image of $C(X)$. In either case, $\phi[C(X)]$ is not contained in $M_{F}$. Now then, if $y \notin F, \quad M_{F}-M_{\nu} \neq \varnothing$ and $\phi f \in M_{P}-M_{y}$ for some $f \in C(X)$. Hence $\phi f(y) \notin \mathrm{Cl}(\phi f[F])$, i.e., $\phi[C(X)]$ separates points and closed sets.

$(2.2 .3) \Rightarrow(2.2 .4)$. Consider $\phi 1$ and suppose there exists a point $y \in Y$ such that $\phi 1(y)=0$. Then for any $f \in C(X), \phi f(y)=[\phi f(y)][\phi 1(y)]=0$ which implies $\phi[C(X)] \subset M_{y}$, a contradiction since the image of $C(X)$ is contained in no $\delta$-real ideal. Hence $\phi 1(y)=1$ for every $y \in Y$, i.e., $\phi 1=1$. By induction, it follows that $\phi \boldsymbol{n}=\boldsymbol{n}$ for every positive integer $n$ and from this it follows that $\phi \boldsymbol{r}=\boldsymbol{r}$ for every rational number $r$. Using the fact that the rationals are dense in $R$ and that $\phi$ is also a lattice homomorphism, it can be shown that $\phi k=k$ for every real number $k$.

(2.2.4) $\Rightarrow(2.2 .1)$. Suppose $g \in C(Y)$ and $y \notin Z(g)$. Then since $\phi[C(X)]$ separates points and closed sets, there is a function $f \in C(X)$ such that $k=\phi f(y) \notin \operatorname{Cl}(\phi f[Z(g)])$. Let $f_{1}=f-k$. Since $\phi$ is the identity mapping on constant functions, $\phi f_{1}(y)=0$ and $\phi f_{1}(y)$ $\notin \mathrm{Cl}\left(\phi f_{1}[Z(g)]\right)$. Hence there exists a positive number $\epsilon$ such that

4. Mrowka pointed out that (2.2.4) is equivalent to (2.2.1). 
$\left|\phi f_{1}(p)\right|>\epsilon$ for $p \in Z(g)$. Now let $f_{2}=0 \vee\left[\epsilon-\left|f_{1}\right|\right]$. Since $\phi\left|f_{1}\right|=\left|\phi f_{1}\right|$, $\phi f_{2}(y) \neq 0$ and $Z(g) \subset Z\left(\phi f_{2}\right)$.

From Theorems (2.1) and (2.2), we get

Theorem (2.3). Let $X$ and $Y$ be $Q$-spaces. A homomorphism from $C(X)$ into $C(Y)$ is a $\delta$-homomorphism if and only if there exists a homeomorphism $h$ from $Y$ into $X$ such that $\phi f=f \circ h$ for all $f \in C(X)$.

The following lemma is needed.

Lemma (2.4). Let $X$ and $Y$ be topological spaces and $h$ a continuous function from $Y$ into $X$. Define a homomorphism $\phi$ from $C(X)$ into $C(Y)$ by $\phi f=f \circ h$. Then for real ideals $M_{x}$ and $M_{y}$ of $C(X)$ and $C(Y)$, respectively, $\phi\left[M_{x}\right] \subset M_{y}$ if and only if $h(y)=x$.

Proof Suppose $h(y)=x$. Then for $f \in M_{x}, \phi f(y)=f(h(y))=f(x)=0$. Thus $\phi\left[M_{x}\right] \subset M_{y}$. Conversely, suppose $\phi\left[M_{x}\right] \subset M_{y}$ and let $f \in C(X)$. Then $f-\boldsymbol{f}(\mathbf{x}) \in M_{x}$ and $\phi f-f(x) \in M_{y}$. Hence $f(h(y))=\phi f(y)=f(x)$ for every $f \in C(X)$ which implies that $h(y)=x$.

Theorem (2.5). Let $X$ and $Y$ be $Q$-spaces. A homomorphism from $C(X)$ into $C(Y)$ is a $\delta F$-homomorphism if and only if there exists a homeomorphism $h$ from $Y$ into $X$ such that $\phi f=f \circ h$ for all $f \in C(X)$, and $h[Y]$ is a closed subset of $X$.

Proof (Sufficiency). Let $M$ be a real ideal of $C(X)$ which contains the kernel, $K(\phi)$, of $\phi$. Then $M=M_{x}$ for some $x \in X$. Moreover, since $h[Y]$ is closed, $x \in h[Y]$ since otherwise there would exist a function $f \in C(X)$ vanishing on $h[Y]$ but not at $x$. This would imply $f \in K(\phi)$ $-M_{x}$, a contradiction. Then $x=h(y)$ for some $y \in Y$ and by the previous lemma, $\phi\left[M_{x}\right] \subset M_{y}$, that is, the image of $M$ is subreal in $C(Y)$.

(Necessity) Now suppose $\phi$ is a $\delta F$-homomorphism. By Theorem (2.3), there is a homeomorphism $h$ from $Y$ into $X$ such that $\phi f=f \circ h$ for all $f \in C(X)$. It remains to show that $h[Y]$ is a closed subset of $X$. Choose $x \in \mathrm{Cl}_{X}(h[Y])=F$. Then $K(\phi)=M_{F}$ and $M_{F} \subset M_{x}$. Therefore there is a real ideal $M_{y}$ of $C(Y)$ such that $\phi\left[M_{x}\right] \subset M_{y}$, which, by Lemma (2.4), implies $x=h(y)$. Thus $x \in h[Y]$ and we conclude that $h[Y]=\mathrm{Cl}_{X}(h[Y])$.

Theorem (2.6). Let $X$ and $Y$ be $Q$-spaces. A homomorphism $\phi$ from $C(X)$ into $C(Y)$ is a $\delta G$-homomorphism if and only if there exists a homeomorphism $h$ from $Y$ into $X$ such that $\phi f=f \circ h$ for all $f \in C(X)$, and $h[Y]$ is an open subset of $X$.

Proof (Sufficiency). Let $M_{x}$ be a real ideal of $C(X)$ whose image is subreal in $C(Y)$. Hence for some $y \in Y, \phi\left[M_{x}\right] \subset M_{y}$. Again using 
Lemma (2.4), we have $h(y)=x$. Then $x \notin X-h[Y]$ which is a closed subset of $X$ and there is an $f \in C(X)$ which vanishes on $X-h[Y]$ but not at $x$. Then $f \notin M_{x}$ and it follows that the image of every real ideal not containing $f$ is subreal.

(Necessity) Now suppose $\phi$ is a $\delta G$-homomorphism and $x \in h[Y]$. Then $x=h(y)$ for some $y \in Y$ and $\phi\left[M_{x}\right] \subset M_{y}$. Therefore there exists a function $f \in C(X)$ such that $f \notin M_{x}$ and the image of every real ideal not containing $f$ is subreal. From this it follows that $x \in[X-Z(f)]$ $C h[Y]$ which in turn implies that $h[Y]$ is open.

Finally, we note that if $h$ is a continuous function from $Y$ into $X$, the homomorphism $\phi$ given by $\phi f=f \circ h$ is an isomorphism if and only if $h[Y]$ is dense in $X$. This, in conjunction with Theorem (2.3), gives:

Theorem (2.7). Let $X$ and $Y$ be $Q$-spaces. $A$ homomorphism $\phi$ from $C(X)$ into $C(Y)$ is a $\delta$-isomorphism if and only if there exists a homeomorphism $h$ from $Y$ into $X$ such that $\phi f=f \circ h$ for all $f \in C(X)$ and $h[Y]$ is a dense subset of $X$.

\section{Bibliography}

1. L. Gillman and M. Jerison, Rings of continuous functions, Van Nostrand, New York, 1960.

2. E. Hewitt, Rings of real-valued continuous functions. I, Trans. Amer. Math. Soc. 64 (1948), 45-99.

3. J. L. Kelley, General topology, Van Nostrand, New York, 1955.

Pennsylvania State University and

State University of New York at Buffalo 